\title{
Technology Acceptance Model in Evaluation of Telematics Technologies Utilization in Road Transportation
}

\author{
Wojciech Zalewski ${ }^{1,}{ }^{*}$, Magdalena Osińska ${ }^{2}$ and Mirosława Żurek ${ }^{3}$
}

1. Department of Logistics, Nicolaus Copernicus University in Torun, Gagarina st. 13A, 87-100 Torun, Po-
land; email: w.zalewski@umk.pl
2. Department of Economics, Nicolaus Copernicus University in Torun, Gagarina st. 13A, 87-100 Torun, Po-
land; email: emo@umk.pl
3. Commercial industry; mirka.zurek@gmail.com
* Correspondence: w.zalewski@umk.pl

\begin{abstract}
The purpose of this paper is to evaluate the acceptance and the utilization of GPS/GPRS-based telematics technology in road transport companies registered in Poland. Telematics technologies are essential for management of energy saving and emissions reduction in road transport. It is in line with the European Union policy of sustainable transportation. The evaluation is based on a survey designed and carried out in 2020. The issues concerning the scope of telematics systems utilization as well as the internal and external factors affecting their use are analysed. The methodology is based on Technology Acceptance Model (TAM) and Structural Equation Modelling (SEM). The results are checked for robustness. Based on the results, it can be reasoned that as a result of the COVID19 pandemic, the companies started to use telematics systems more widely than they did before. Furthermore, the companies employing more people recognize the higher usefulness of telematics systems and are motivated to have the systems more than smaller enterprises; however, TAMs estimated separately for small and medium-sized enterprises did not reveal any significant differences in the parameter estimates.
\end{abstract}

Keywords: econometrics, road transportation, telematics, survey data, fuel consumption

\section{Introduction}

The purpose of this paper is to evaluate the acceptance and the actual utilisation of GPS/GPRS-based telematics technology in road transport companies registered in Poland. Since 1992 the European Commission puts much attention to transport policy creation, mainly to transport sustainability. A series of white books were issued introducing new targets in the analyzed period. In the white book projected for the years 2011-2020 [1], the European Commission assumed that the highest priority is assigned to the unified European transport area creation till 2050. A new European transport system, being competitive and resource-efficient, should significantly reduce conventional fuel use and $\mathrm{CO} 2$ emission and utilize a new generation of fuels [2]. For road transport, the most urgent targets are: to produce a new generation of cars and trucks equipped in modern EUR6 and EUR7 engines, to apply solutions based on intelligent transport systems [3-4], and to utilize telematics solutions to control - among others - fuel use and exhaust fumes emission [5].

The motivation for current research comes from the results obtained in a study carried out in 2019 on the extent of telematics equipment utilization to support transport and management processes. The studies [6,7] revealed that on the one hand the solutions are omnipresent, but on the other hand the offered functionalities are used only to a 
certain degree, including but not limited to supporting transport and management processes. That is why another survey was designed and carried out in 2020. It was aimed to answer the question concerning the scope of telematics systems utilisation and the internal and external factors affecting their use. Internal factors mean the factors that originate from the company, while the external ones are the stimuli from the company's environment. As a result of COVID19 pandemic, many companies shifted to remote work and all parties: contractors, clients, and clerks have got used to electronic documents. That is why the scale of using systems integrated with telematics systems in road transport has increased significantly. Still, the answer to whether internal factors provided sufficient support for the use of telematics-management tools will be possible only after analysis.

The terminology used in the study resulted from the structure of the Technology Acceptance Model [8], (TAM). It enables the identification of the cause-and-effect relationships between the perceived usability and the ease of telematics systems' use, as well as the attitude towards the use and the actual use. The possibility of identifying direct and indirect cause-and-effect relationships and extending the model with subsequent factors that affect the telematics systems use are the advantage of the TAM. Regarding the fact that most road transport companies are micro-companies or small and medium-sized enterprises (SMEs), the assumption was made in the study that the discussed cause-and-effect relationships can depend on the company size. According to research on SMEs, it can be assumed that due to limited resources there are significant differences in SMEs management methods compared to big companies that most management theories apply to [9-10]. An important indication comes from the findings reported by Turner, Ledwith and Kelly [11]. They examined 118 respondent companies, divided into micro-companies and SMEs, and hi-tech, low-tech, and service industries. They found that companies of all sizes spend roughly the same proportion of their turnover on projects, but the smaller the company, the smaller its projects are, and the less it uses project management and its tools. Strangely enough, hi-tech companies spend less on projects than low-tech or service companies, but have more extensive projects and use project management to a greater extent.

Taking these findings into account and implementing the road transport industry's characteristics, we assumed that micro and small transport enterprises are less likely to benefit from telematics systems due to lower knowledge of their application in transport activity management. Therefore we implemented the enterprise sizes in the Technology Acceptance Model.

Our paper contributes to the existing literature in the novel way of examining the degree of telematics integrated technologies acceptance and utilisation by road transport enterprises registered in Poland but operating in the entire Europe. To the best of our knowledge, there is no similar research in any publications. Typically a single user (an individual) is asked to demonstrate or compare their motivation to use a given application. Here, we focus on the enterprise's management level. Thus managers are responsible for using telematics devices and integrated packages for the operational management of the company. So an enterprise was defined as a microunit. We do not consider any particular product. We analyse how much the enterprises realise that they benefit from this 
kind of support. Since Polish road transport sector (along with the German and British ones) is among the most significant transport stakeholders in Europe and has the newest generation of trucks and semitrailers, the findings and conclusions can be easily extended [12]. Another incentive to take advantage of the new solutions is that the questionnaires were collected during the first phase of COVID19 pandemic. New organisational and legal circumstances contributed to an increase in the popularity of hi-tech devices. In the study, we examined 500 enterprises which responded to the questions in June-July, 2020.

\section{Materials and Methods}

In 1985 Davis presented The Technology Acceptance Model (TAM) concept [8]. It was based on the assumption that a decision about using new information technology is the user's behavioural reaction that can be explained or even predicted based on the user's motivation; the motivation is in turn affected directly by external stimuli resulting from the technology's current features and capabilities, the user's characteristics and organisational factors [13]. The technology acceptance is meant as a will, demonstrated in the users' group, to use the information technology in order to implement the tasks that the technology was designed to support [14].

Based on these assumptions, Davis extended his conceptual model to the version shown in Figure 1.

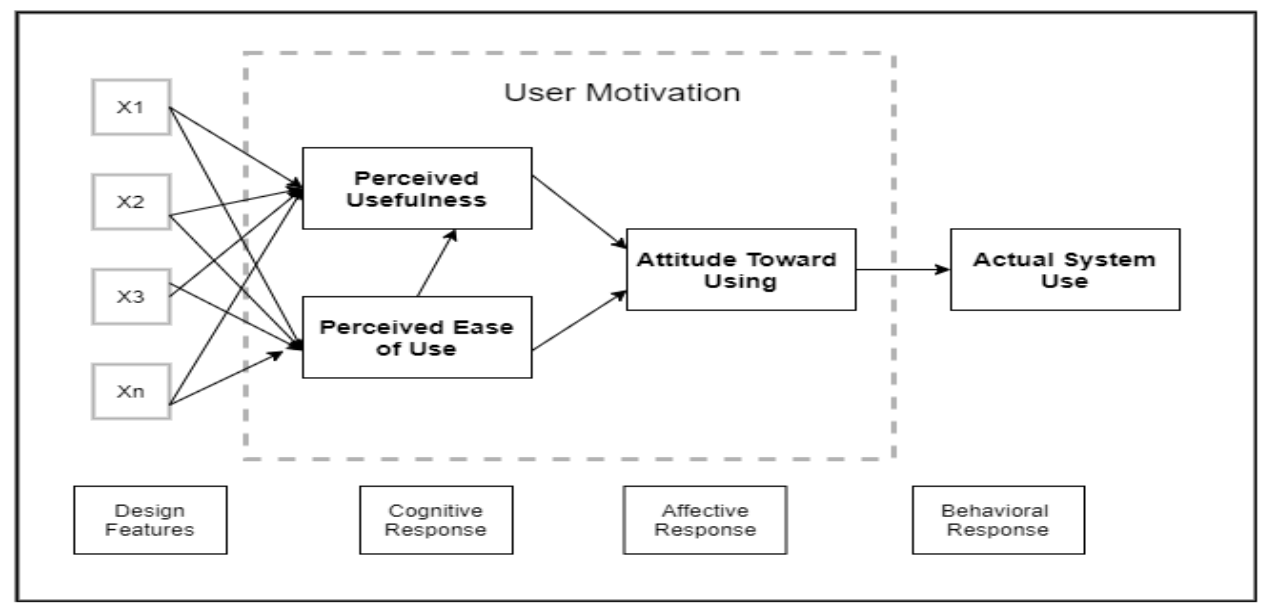

Fig. 1. Original Technology Acceptance Model diagram developed by Davis

He proposed that the user's motivations can be explained with the three main factors: Perceived Ease of Use (PEU), Perceived Usefulness (PU) and Attitude Toward Using (ATU), which are latent variables. He assumed that the user's attitude toward technology is the primary determinant of the decision to either use or reject the system. He also claimed that the user's attitude is affected by two convictions: Perceived Usefulness and Perceived Ease of Use, whereby the latter directly influences the Perceived Usefulness. Moreover, it was assumed that the system design features (marked as $\mathrm{X} 1, \mathrm{X} 2, \mathrm{X} 3$ to $\mathrm{Xn}$ in Fig. 1), also referred to as external variables, directly impact the two convictions. Davis intended to develop a simple, theoretically justified model capable of explaining the factors which determine the use of computer systems in general, i.e. for different users' end groups and different system types. The TAM is then a theoretical base which explains how different factors affect the convictions, attitudes and intentions [15]. 
The original model developed by Davis in 1985 has been subjected to many transformations and extensions. Venkatesh and Davis [13], proposed the TAM2 model, which provided a more accurate and detailed explanation of the reasons why some users accepted the use of the particular system or technology in relation to time, namely before the implementation, one month after the implementation and three months after the implementation.

Venkatesh and Bala [16], combined TAM2 and the Perceived Ease of Use determinants model [17], to develop an integrated technology acceptance model known as TAM3. In this model, the authors completed the catalogue of the system/technology Ease of Use determinants.

Further studies carried out to modify the TAM model and adapt it to the changing reality led to the development - by Venketesh et al. - of a uniform theory of technology acceptance and use, which was the base for The Theory of Acceptance and Use of Technology (UTAUT) model [18].

There is an abundance of papers devoted to TAM and its modifications. Analysing two databases, i.e. Web of Science and Scopus for the period between 1997 and 2020, and searching according to the paper title, abstract, keywords and the text content for the "Technology Acceptance Model" phrase, we get the search result of 18,845 papers in Web of Science and 14,429 papers in Scopus.

It shall be concluded that the number of papers and their topics are highly diversified. Many journal articles related to GPS/GPRS telematics systems and Global Navigation Satellite System (GNSS) refer to technical description and simulation of the systems. Humphreys et al. [19], examined effectiveness of GNSS system for high-level accuracy in positioning objects in a deep urban setting unaided by complementary sensors. Kassas et al. [20], provided a framework for ground vehicle localization that uses cellular signals of opportunity, a digital map, an inertial measurement unit, and a Global Navigation Satellite System (GNSS) receiver. This framework aims to enable localization in an urban environment where GNSS signals could be unusable or unreliable. The results helped much reducing localization errors. Fernandez-Hernandez et al. [21], focused on a high-accuracy service (HAS) provided by the Global Navigation Satellite System.

An analysis of the journals' databases indicates that despite high popularity and many studies carried out on the technology acceptance, only a few of them were devoted to the use of telematics as a modern technology discipline. There are no papers on the acceptance of telematics technologies in road transport. Another analysis of Scopus and Web of Science databases, using a similar search method but based on the phrases "Technology Acceptance Model in Telematics" or "Technology Acceptance Model \& Telematics Systems \& Road Transport", rendered only 11 papers in Scopus and 54 ones in Web of Science. The papers' content analysis revealed that they did not apply directly to the issues related to GPS/GPRS-based telematics technologies used by road transport enterprises, nevertheless they contributed in the assessment of individual perception of innovative solutions in transportation.

Chen and Chen [22], analysed factors affecting the travellers' intentions related to the use of GPS products and systems installed in the vehicles. The survey questionnaire 
was designed based on the Technology Acceptance Model (TAM) and unique properties of GPS equipment. The results revealed that the Perceived Ease of Use had a significant and positive impact on the Perceived Usefulness. Every Perceived Usefulness, Perceivable Pleasure of Use and Ease of Use have a significant positive influence on the Attitude Towards Using. The Attitude Toward Using significantly affects the behavioural intention and will to use. Personal innovativeness moderates the relationship between the attitude and behavioural intention.

Park et al. [23], carried out a study on a population of 1,011 participants and using the Structural Equations Model (SEM) to establish the technological acceptance criteria for the applied mobile map services. The study results revealed that the Perceived Mobility and the Perceived Location Accuracy significantly affected the users' acceptance of their intention to use mobile map services via computer equipment. An increase in the Perceived Mobility positively affected the Perceived Usefulness and Ease of Use of the services. Moreover, the study revealed a more substantial influence of the approach on the behavioural intention than the Perceived Usefulness.

Nordhof S. et al. [24], reported on the study of social acceptance for using partially automated and autonomous passenger vehicles. A survey was carried out in a population of 9,118 drivers in eight European countries. $71.06 \%$ of the respondents decided that partially automated cars are easy to drive, and $28.03 \%$ of the study participants intend to buy an automated car when it is available. The Unified Theory of Acceptance and Use of Technology (UTAUT2) was used to investigate the effects of the capacity and expected effort, social impact, facilitating factors and hedonic motivation on the behavioural intention to use partially automated vehicles.

Recently, Yang et al. [25], have proposed an integrated technology acceptance model to investigate the factors which affect drivers' intention to use mobile navigation applications, which can also apply to road transport. The study was carried out on a population of 384 drivers. The vital element included optimising mobile applications by programmers so that apps did not distract the driver's attention while driving a vehicle.

\section{Results}

\subsection{TAM in assessment of telematics acceptance by road transport enterprises}

In the paper, we constructed The Technology Acceptance Model and proposed its modification taking into account the original Davis's motivation. As we decided to study the companies from the road transport sector, we assumed that our targets are defined as these companies' managers and owners. Thus, we can assume that they possess broader knowledge than the users of the typically employed systems in these companies. So the model construction was projected at the management level. Therefore the Perceived Use was defined as the Perceived Usefulness of information coming from telematics systems for the operational management of the transport processes observed in the company. Consequently, the Perceived Use (PU) latent variable was described by increasing the system's efficiency in the following areas: the number of orders, timeliness, market confidence, costs control and profitability of orders. The Perceived Ease of Use (PEU) was defined by retracing the routes, truck punctuality in the sender's and receiver's locations, 
time delays reporting, driving time analysis and fuel consumption. For fuel consumption analysis (variable $x 5$ in Appendix A), transport managers most often indicated number 7 in the seventh-point Likert scale, exhibiting great attention for fuel consumption control. It also evidences that users perceive the fuel consumption control as easy with the telematics systems. The perceived ease of use (PEU) is, here, related to the standard scope of analysis in transport activity. Moreover, it was assumed that telematics system enables preparing analysis in more precise, convenient and fast way than standard office tools. It can be noticed that PU and PEU are defined by internal features that come from the enterprise. The next variable - Attitude Toward Use (ATU) covers external features from the enterprise's environment. The following characteristics defined the Attitude Toward Use: a significance of information coming from telematics systems, safety of data collected in the telematics system, and level of autonomy in making decisions about using a telematics system. The latter concerns the links in the supply chains where a given transport enterprise operates. The actual use was observed as practical use of the system.

The observed characteristics are thus closer to the manager's observations in the enterprise than individual psychological features, as it was reported by Davis [8], and former papers utilising the TAM theory. A cognitive feature such as the Perceived Use is thus related to the manager's Perceived Usefulness. The Perceived Ease of Use could be reported by managers as well as individual employees who use the system. The Attitudes Towards Use are based on the managers' perception concerning facts and belief in the system's data protection power. In this context we perceive managers as the rational (or informed) power of the enterprise, taking into consideration both advantages and costs of telematics systems utilisation. Furthermore, the requirements from the company's environment (contractors) may increase interest in employing a broader scope of telematics.

Previous studies [6-7] revealed that enterprises applied telematics systems to track vehicle routes. Other advantages of the system included the determination of the drivers' working time in a given 24-hour driving period, analysing whether the driver will be able to arrive at the destination within the pre-determined time slot for loading or unloading, analysing weekly and bi-weekly periods, compatibility of telematics systems with cargo exchanges and client applications, and compatibility with other onboard devices, e.g. refrigeration units.

Recently, when the COVID19 pandemic has changed the reality, entrepreneurs became aware that telematics offers more than truck tracing. That is why the study can be treated as reliable because the managers realised the advantages of the system. One issue needs to be explained, though. The enterprises use telematics systems for different periods, ranging from several months to several years. We assumed that they reported the system's usefulness as they had assessed it for the survey time. In order to prepare the Technology Acceptance Model for telematics, we developed a survey questionnaire. The respective questions are described in the Appendix A.

\subsection{Data characterictics}


The survey, aimed to identify the factors of telematics technology acceptance, was conducted in June-July, 2020 among 500 randomly selected road transport enterprises that carry goods using the CATI method. The surveyed units were defined for the material criterion, i.e. enterprises providing road transport services, and territorial criterion, i.e. enterprises whose business activity is registered in Poland. The survey was carried out based on a sample of enterprises from the databases of the members of the Association of International Road Carriers in Poland. Consequently, the enterprises were selected from a population of 4,500 companies. The fact that decision-makers took part in the study (they were managers competent in the scope of the answers provided) was a vital aspect of the study. The number of 500 respondents was determined by the statistical assumption that the maximum error rate in the estimation would not exceed $5 \%$ [26]. The structure of the sample is presented in Table I.

Table I. Structure of the studies sample

\begin{tabular}{|c|c|c|c|}
\hline Participation of enterprises by the form of conducted activity & $\%$ & $\begin{array}{l}\text { Share of enterprises by the number of } \\
\text { employees }\end{array}$ & $\%$ \\
\hline Sole proprietorship & 2.20 & 1 to 9 & 6.60 \\
\hline Limited liability company & 80.80 & 10 to 49 & 56.00 \\
\hline General partnership & 13.80 & 50 to 249 & 32.40 \\
\hline Joint-stock company & 2.20 & Over 250 & 5.00 \\
\hline Other (limited partnership) & 1.40 & & \\
\hline Participation of enterprises by the homogeneity of the conducted business activity & $\%$ & The range of services provided & $\%$ \\
\hline At least $80 \%$ of revenues generated from transport activities & 74.20 & International & 66.20 \\
\hline Less than $80 \%$ of revenues generated from transport activities & 25.80 & Domestic & 23.40 \\
\hline \multirow[t]{2}{*}{ Participation of enterprises by experience in running a business } & $\%$ & Regional & 7.10 \\
\hline & & Local & 3.30 \\
\hline More than 10 years & 95.00 & & \\
\hline From 5 up to 10 years & 2.80 & & \\
\hline From 3 up to 5 years & 1.20 & & \\
\hline Up to 3 years & 1.00 & & \\
\hline
\end{tabular}

The data presented in Table 1 reveal that the sample of 500 enterprises selected for the study roughly reflects the structure of transport enterprises providing domestic and international transport services, in line with the data presented in the report GITD [27], . An analysis of the survey data revealed that all enterprises participating in the survey had vehicles with telematics systems installed. In total, all surveyed enterprises owned 24,975 vehicles, $97.3 \%$ of which were equipped with a telematics system (24,304 vehicles). Enterprises used a wide range of different telematics products, such as Tom Tom (20\%), Navi Expert (17\%), MIX Telematics (13\%), Fleetmatics (13\%), however no significant dominance of any product was observed.

\subsection{Estimation results}

A. Measurement constructs in the TAM model 
The list of all survey questionnaire questions analysed in the paper is included in Appendix A. A relevant designation of the variable $x_{i}, i=1 \ldots .14$ was assigned to the questions used for the latent variables definition and construction. According to the definition proposed by Davis, the usefulness of the telematics technologies used in the enterprise should reflect the degree of the users' conviction that the technologies will improve the results of the users' work or help achieve much higher operating capacity owing to the use of specific technology. The Perceived Usefulness of the telematics system was explained in the study by determining how it improves the enterprise's performance by increasing the system's efficiency in the following areas: a number of orders, timeliness, market confidence, costs control and profitability of orders. The measurement method of the Perceived Usefulness (PU) and Perceived Ease of Use (PEU) variables was discussed in the previous section. It was assumed in the study that the Attitude Toward Using (ATU) for telematics systems must not be identical only with the concept of autonomous, rather than forced decision concerning their implementation in the company. That is why this factor includes the safety of telematics systems perceived by the users and the awareness of the system's usefulness for the company operation. The observable variables for this factor were marked as $\mathrm{x}_{11}, \mathrm{x}_{12}$ and $\mathrm{x}_{13}$. Only the resultant variable in the TAM - the Actual System Use (USE) - was reflected directly in the survey questionnaire, in the question applying to the degree of using the telematics system in the company $\left(\mathrm{x}_{14}\right)$. It is the only observable variable.

All analysed factors, which are latent variables, included in the TAM, i.e., PU, PEU and ATU were verified for the scale reliability. The values of Cronbach's alpha (CA) statistics for PEU and PU are much higher than the recommended value of 0.7, which means very good scale reliability [28]. The CA statistics values are higher than the required level, only for the ATU variable the value of the statistics is slightly lower. Still, the difference is small and amounts to ca. $6 \%$ of the recommended value. That is why the ATU variable was included further in the analysis.

Table II. Summary of latent variables and reliability statistics (AC)

\begin{tabular}{lcc}
\hline \hline Latent variable & Variables & Cronbach's alpha statistics (AC) \\
\hline PEU (Perceived Ease of Use) & $\mathrm{x}_{1}, \mathrm{x}_{2}, \mathrm{x}_{3}, \mathrm{x}_{4}, \mathrm{x}_{5}$, & 0.847 \\
PU (Perceived Usefulness) & $\mathrm{x}_{6}, \mathrm{x}_{7}, \mathrm{x}_{8}, \mathrm{x}_{9}, \mathrm{x}_{10}$ & 0.847 \\
ATU (Attitude Toward Using) & $\mathrm{x}_{11}, \mathrm{x}_{12}, \mathrm{x}_{13}$ & 0.661 \\
USE (Actual Use of the System) & $\mathrm{x}_{14}$ & NA \\
\hline \hline
\end{tabular}

\section{B. Estimated TAMs}

Structural Equation Modeling (SEM) methodology was used to determine the relationship between the Perceived Usefulness (PU), Perceived Ease of Use (PEU), Attitude Toward Using (ATU) and Actual System Use (USE) according to TAM. The models enable the analysis of cause-and-effect relationships between the latent variables, which cannot be measured directly [29-31].

Two TAMs were estimated in this paper - a classical and extended TAM in which an additional variable was included. The company size was the new variable, measured directly with the workforce number (EMP variable). Both models were estimated in the 
SPSS AMOS v.16 package with the maximum likelihood (ML) method. The significance level of 0.05 was assumed for statistical analysis. Above these, the classical TAM was also estimated in two variants: for small enterprises (headcount up to 49 people) and medium-sized enterprises (headcount of 50 or more people). The estimation results for all TAM variants are summarised in Table III.

Table III. Estimated TAM models

\begin{tabular}{|c|c|c|c|}
\hline Relations & Estimate & S.E. & p-value \\
\hline \multicolumn{4}{|c|}{ Classic TAM model (total sample) } \\
\hline $\mathrm{PEU} \rightarrow \mathrm{PU}$ & 0.526 & 0.050 & 0.000 \\
\hline $\mathrm{PEU} \rightarrow \mathrm{ATU}$ & -0.077 & 0.039 & 0.258 \\
\hline $\mathrm{PU} \rightarrow \mathrm{ATU}$ & 0.374 & 0.048 & 0.000 \\
\hline ATU $\rightarrow$ USE & 0.763 & 0.140 & 0.000 \\
\hline \multicolumn{4}{|c|}{$\mathrm{CMIN} / \mathrm{DF}=2.023 \mathrm{IFI}=0.969 \mathrm{RMSEA}=0.045$} \\
\hline \multicolumn{4}{|c|}{ Extended TAM model (total sample) } \\
\hline $\mathrm{EMP} \rightarrow \mathrm{PU}$ & 0.147 & 0.000 & 0.000 \\
\hline $\mathrm{EMP} \rightarrow \mathrm{ATU}$ & 0.174 & 0.000 & 0.000 \\
\hline $\mathrm{PEU} \rightarrow \mathrm{PU}$ & 0.523 & 0.050 & 0.000 \\
\hline $\mathrm{PEU} \rightarrow \mathrm{ATU}$ & -0.065 & 0.039 & 0.340 \\
\hline $\mathrm{PU} \rightarrow \mathrm{ATU}$ & 0.338 & 0.048 & 0.000 \\
\hline ATU $\rightarrow$ USE & 0.762 & 0.138 & 0.000 \\
\hline \multicolumn{4}{|c|}{$\mathrm{CMIN} / \mathrm{DF}=1.917 \mathrm{IFI}=0.968 \mathrm{RMSEA}=0.043$} \\
\hline \multicolumn{4}{|c|}{ Classic TAM model for micro and small enterprises } \\
\hline $\mathrm{PEU} \rightarrow \mathrm{PU}$ & 0.513 & 0.063 & 0.000 \\
\hline $\mathrm{PEU} \rightarrow \mathrm{ATU}$ & -0.036 & 0.045 & 0.670 \\
\hline $\mathrm{PU} \rightarrow \mathrm{ATU}$ & 0.306 & 0.055 & 0.001 \\
\hline ATU $\rightarrow$ USE & 0.756 & 0.211 & 0.000 \\
\hline \multicolumn{4}{|c|}{$\mathrm{CMIN} / \mathrm{DF}=1.824 \mathrm{IFI}=0.961 \mathrm{RMSEA}=0.052$} \\
\hline \multicolumn{4}{|c|}{ Classic TAM model for medium-sized enterprises } \\
\hline $\mathrm{PEU} \rightarrow \mathrm{PU}$ & 0.562 & 0.085 & 0.000 \\
\hline $\mathrm{PEU} \rightarrow \mathrm{ATU}$ & -0.132 & 0.066 & 0.255 \\
\hline $\mathrm{PU} \rightarrow \mathrm{ATU}$ & 0.437 & 0.083 & 0.000 \\
\hline $\mathrm{ATU} \rightarrow \mathrm{USE}$ & 0.762 & 0.198 & 0.000 \\
\hline & $A=0.053$ & & \\
\hline
\end{tabular}

The results obtained for the total sample of enterprises confirm the statistically significant influence of the Perceived Ease of Use (PEU) on the Perceived Usefulness (PU), the Perceived Usefulness on the Attitude Toward Using (ATU) and the Attitude Toward Using on the Actual Use of the System (USE). It means that the less complicated the system (PEU), the higher its Perceived Usefulness (PU) is. As the Perceived Usefulness (PU) increases, the greater the motivation to use it (ATU) is, which contributes to the 
actual broader use of telematics systems (USE). The relationship between the Perceived Ease of Use (PEU) and Attitude Toward Using (ATU) is statistically insignificant.

Based on the results obtained for the extended TAM it can be reasoned that the companies that employ more people (EMP) recognise higher usefulness of telematics systems (PU) and are motivated to have the systems (ATU) more than smaller enterprises. The results comply with the authors' intuition and experience in the field. However, TAMs estimated for small and medium-sized enterprises did not reveal any significant differences in the parameter estimates. The only major difference in the parameter evaluation for both groups was observed for the PU $\rightarrow$ ATU relationship and amounted to 0.131 (0.437-0.306 difference). The calculated statistics $t=1.316(p=0.188)$ does not allow for evaluating the difference as statistically significant [32]. It is the stability of the parameter estimates across the samples used in research that matters.

The CMIN/DF statistics values lower than 2, IFI higher than 0.95, and RMSEA less than 0.05 are a testimony of a perfect adaptation of the models to the data [28]. Only for models based on subgroups defined by the number of employees, the value of the RMSEA coefficient is slightly higher. For both groups, it is lower than 0.055 , so it confirms that the model is well fitted to the data.

\subsection{Robustness check}

Despite the correct statistics confirming the model's quality, the results were additionally verified. A bootstrap procedure using the maximum likelihood (ML) estimator was employed to re-estimate the model parameters. The procedure was used for the model estimated based on the total sample. The bootstrap based on 5,000 samples helped calculate the parameter bias and standard errors of the biases, and determine the bias-corrected confidence intervals of $95 \%$ [33]. The results for internal TAMs are summarised in Table IV.

Table IV. Results of TAMs estimation using bootstrap

\begin{tabular}{|c|c|c|c|c|c|c|}
\hline Relations & Estimate & Bias & S.E. Bias & Lower & Upper & $\mathrm{p}$ value \\
\hline \multicolumn{7}{|c|}{ Classic TAM model (total sample) } \\
\hline $\mathrm{PEU} \rightarrow \mathrm{PU}$ & 0.526 & 0.004 & 0.001 & 0.441 & 0.614 & 0.000 \\
\hline $\mathrm{PEU} \rightarrow \mathrm{ATU}$ & -0.077 & 0.000 & 0.001 & -0.213 & 0.054 & 0.315 \\
\hline $\mathrm{PU} \rightarrow \mathrm{ATU}$ & 0.374 & -0.001 & 0.001 & 0.232 & 0.517 & 0.000 \\
\hline ATU $\rightarrow$ USE & 0.763 & -0.005 & 0.001 & 0.698 & 0.810 & 0.000 \\
\hline \multicolumn{7}{|c|}{ Extended TAM model (total sample) } \\
\hline $\mathrm{EMP} \rightarrow \mathrm{PU}$ & 0.147 & 0.000 & 0.000 & 0.087 & 0.216 & 0.001 \\
\hline $\mathrm{EMP} \rightarrow \mathrm{ATU}$ & 0.174 & 0.000 & 0.000 & 0.105 & 0.236 & 0.001 \\
\hline $\mathrm{PEU} \rightarrow \mathrm{PU}$ & 0.523 & -0.003 & 0.001 & 0.436 & 0.609 & 0.000 \\
\hline $\mathrm{PEU} \rightarrow \mathrm{ATU}$ & -0.065 & 0.000 & 0.001 & -0.198 & 0.061 & 0.371 \\
\hline $\mathrm{PU} \rightarrow \mathrm{ATU}$ & 0.338 & -0.001 & 0.001 & 0.203 & 0.487 & 0.000 \\
\hline ATU $\rightarrow$ USE & 0.762 & 0.021 & 0.003 & 0.697 & 0.808 & 0.000 \\
\hline
\end{tabular}

Based on the results, it can be observed that the parameters reflecting the influence of the employment (EMP) variable on the PU and ATU for the extended model were 
non-biased. The parameter bias value is assumed as statistically insignificant when the standard bias error is greater than the bias itself (absolute value) [34]. It is valid for the PEU $\rightarrow$ ATU relationship in both estimated models. The presence of the model parameters bias does not prejudge a lack of their statistical significance. The confidence intervals corrected with the bias confirm the reliability of the parameters estimated with the maximum likelihood method (PEU $\rightarrow$ PU; PU $\rightarrow$ ATU; ATU $\rightarrow$ USE). The confidence level determined for the PEU $\rightarrow$ ATU relationship includes 0 value in both models, suggesting the parameter's insignificance, analogically to the findings made with the maximum likelihood method. Hence, the ML estimated models' results and verified with the bootstrap enable reliable inference based on the models.

\section{Discussion}

The Technology Acceptance Model is a useful analytical tool for telematics technology acceptance by the road transport sector for two reasons. First and foremost, the model is very well rooted in the behavioural context, and secondly, it is universal, which means it can be used on different analysis levels. The results obtained for the studied enterprises (total sample and divided into micro, small and medium-sized enterprises) confirm a statistically significant influence of the Perceived Ease of Use (PEU) on the Perceived Usefulness (PU), the Perceived Usefulness on the Attitude Toward Using (ATU) and the Attitude Toward Using on the Actual Use of the System (USE). It means that the less complicated the system (PEU), the higher its Perceived Usefulness (PU) is. Simultaneously, as the Perceived Usefulness (PU) increases, the greater the motivation to use it (ATU), which contributes to the actual broader use of telematics systems (USE). The relationship between the Perceived Ease of Use (PEU) and Attitude Toward Using (ATU) is statistically insignificant, which may be attributed to the fact that business entities have been using ICT technologies for quite a long time, and their scope has been extending gradually. Hence, the Perceived Ease of Use may slightly lose its significance versus the Perceived Usefulness as the factor that determines the use of the enterprise's telematics system. Adequate service and support for the institution implementing the system may significantly mitigate the potential difficulty. An analysis of the sensitivity of the results confirmed their stability and reliability.

As was mentioned in the introduction, the area and level of telematics systems application were the subject of previous research carried out in 2019 among road transport companies [7]. The scope and level of telematics solutions advancement in road transport companies were analysed in the research for the barriers to their using and clients' requirements. The results suggested that the barriers to using telematics devices are strongly related to the contractors' requirements, which is linked to the scope of the devices' use by road transport companies. An increase in the scope of telematics device use contributed to a rise in the added value in the enterprises.

Research carried out in 2020 for this study revealed a significant change in the level of telematics device use and motivation to use them. Most respondents $(80 \%)$ claimed that the use of telematics systems had a significant influence on the effectiveness and efficiency of the whole company's operation, contributed to a higher number of orders executed per time unit and more effective use of the driver's working time and increased 
the entrepreneurs' trust in the company. A comparative assessment of the 2019 and 2020 research reveals a substantial change in the perception (attitude), application and use of telematics systems.

The pandemic conditions completely changed the attitude to telematics systems and forced moving many operations to ICT platforms often based on remote solutions. It can be regarded as a positive effect of the pandemic, considered in terms of the Shumpeterian 'creative destruction' [35]. Such solutions seem to be long-lasting, which will cause a significant change in the manager's approach to the employed solutions in a long-term perspective. As Contractor [36], argues after the pandemic business is facing implementing better information-gathering systems, 5G surveillance and monitoring, blockchain and other integration of vendor-buyer computer systems. Looking closer into the European environment, after Brexit the changing transport requirements promote the long-lasting use of the reference solutions [37]. For these reasons the final conclusion is that enterprises using telematics systems are subject of both advantages for vehicle tracing and operational management in the enterprise and external incentives caused by supply chains requirements and unexpected shocks.

\section{Conclusions}

This study's subject matter focused on assessing the acceptance level of GPS/GPRS-based telematics technology by road transport companies. The Technology Acceptance Model (TAM) was used for this purpose. Despite a broad scope of the TAM applications in the original version proposed by Davis in 1985 as well as its numerous modifications, the literature makes no mention of research on the acceptance of telematics technologies linked with road transport management systems. This study fills the gap. Our study proposed using a classical and extended (to include workforce number) TAM; we also estimated TAM models by micro, small, and medium-sized enterprises.

A random sample of 500 road transport companies registered in Poland was used for the study. Considering the scale and range of the transport operations performed by Polish transport companies all over Europe and their similarity for equipment modernity, the scope of operation and size, the companies were selected correctly, which enables making generalisations on the European scale.

The construct of (latent) measurement variables applied to the enterprise level. The obtained empirical results turned out to be stable and cohesive for all estimated models. The following sequence of cause-and-effect relationships was established: PEU $\rightarrow$ PU $\rightarrow$ ATU $\rightarrow$ USE. The estimated models revealed adequate values of statistical indicators and resistance to potential bias.

Comparing the results with the results of research carried out in 2019 indicates that the pandemic effect was observed in 2020, which involved broader use of ICT applications and devices, including telematics ones, by road transport companies. This is a positive and probably long-lasting effect because micro and small enterprises need more time to implement the solutions which entail extra expenditure. A forced implementation combined with an analysis of the operational effectiveness caused both a significant qualitative change in transport service and great attention paid to fuel 
consumption and exhaust fumes emission.

Author Contributions: Conceptualization, W.Z. and M.O.; methodology, W.Z. M.O. MZ; software, M.Z.; validation, M.O. and M.Z.; formal analysis, W.Z..; investigation, W.Z. M.O. MZ.; resources, W.Z.; data curation, W.Z.; writing-original draft preparation, W.Z. M.O. MZ.; writing-review and editing, MO., W.Z..; visualization, M.Z.; supervision, M.O..; project administration, W.Z.; funding acquisition, W.Z.. All authors have read and agreed to the published version of the manuscript.

Funding: This research was funded by financial support by the project Future/05/2020 Sustainable Development and the Society of The Future realized at the Nicolaus Copernicus University in Torun, Gagarina 11, 87-100 Torun, Poland.

Data Availability Statement: if the article is published, the data will be made available from the Nicolaus Copernicus University Repository

Conflicts of Interest: The authors declare no conflict of interest. The funders had no role in the design of the study; in the collection, analyses, or interpretation of data; in the writing of the manuscript, or in the decision to publish the results.

Appendix A

Survey Questionns (Likert Scale 1-7; 1 -no impact, 7 - significant impact

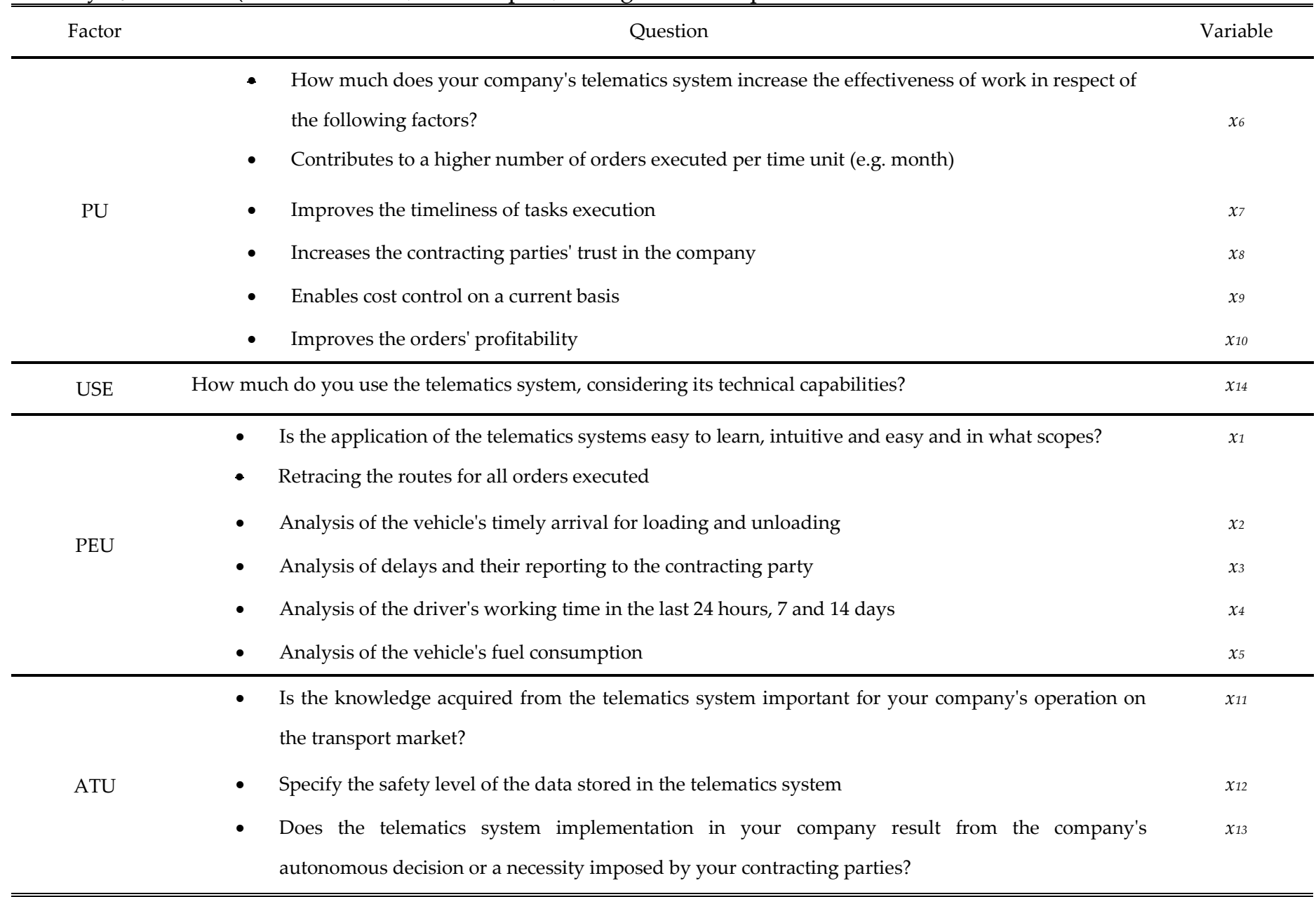

\section{References:}


1. https://ec.europa.eu/transport/sites/transport/files/themes/strategies/doc/2011_white_paper/white-paper-illustrated-brochure e $\underline{\text { n.pdf }}$ (accessed:20.03.2021)

2. https://ec.europa.eu/transparency/regdoc/rep/1/2019/PL/COM-2019-559-F1-PL-MAIN-PART-1.PDF (accessed:20.03.2021)

3. Mishra S., (2014), Intelligent Transportation System, International Journal of Civil Engineering Research. ISSN 2278-3652 Volume 5, Number 4 (2014), pp. 367-372

4. Stawiarska E., Sobczak P., (2018) The Impact of Intelligent Transportation System Implementations on the Sustainable Growth of Passenger Transport in EU, Sustainability, An Open Access Journal from MDPI, 10, 1318; doi:10.3390/su10051318

5. Niebel W., Ebendt R., Kuhns G., (2014), Telematics for the Analysis of Vehicle Fleet Fuel Consumption, in Mikulski J., (Ed.): TST 2014, CCIS 471, pp. 461-468, 2014 (C) Springer-Verlag Berlin Heidelberg; http://link.springer.com/chapter/10.1007/978-3-662-45317-9 49

6. Zalewski, W.; Telematics systems in road transport, Journal of Positive Management, 10(1), 3-20, 2019. http://dx.doi.org/10.12775/JPM.2019.001

7. Zalewski, W.; "Advantages and limitations to application of telematics systems in the road transport industry in Poland", 36th International Business Information Management Association Conference (IBIMA), Granada, Spain, 4-5 November 2020. . ISBN: 978-0-9998551-5-7

8. Davis, F., A technology acceptance model for empirically testing new end-user information systems: theory and results. Cambridge, MA: MIT Press, 1985.

9. Analoui, F. ; Karami, A., Strategic Management in Small and Medium Enterprises, University of Bradford, 2003.

10. Pillania, K.R., Strategic issues in knowledge management in small and medium enterprises, Knowledge Management Research $\mathcal{E}$ Practice, Vol. 6, 334-338), 2008. http://dx.doi.org/10.1057/kmrp.2008.21

11. Turner, J.R.; Ledwith, A.; Kelly, J., Project management in small to medium-sized enterprises: A comparison between firms by size and industry, International Journal of Managing Projects in Business. Vol. 2 No. 2, pp. 282-296, 2009. doi.org/10.1108/17538370910949301

12. EU Transport and figures, (2020), https://op.europa.eu/en/publication-detail/-/publication/ da0cd68e-1fdd-11eb-b57e-01aa75ed71a1, accessd: 02.02.2021. (accessed: 18.03.2021)

13. Venkatesh,V.; F. Davis, F., A theoretical extension of the technology acceptance model: Four longitudinal field studies, Management Science, Vol. 46 No. 2, pp.86-204, 2000. doi.org/10.1287/mnsc.46.2.186.11926

14. Dillon, A.; Morris, M., From "can they" to "will they?": extending usability evaluation to address acceptance, In: Ellen Hoadley, Izak Benbasat eds. Proceedings of the Fourth Americas Conference on Information Systems (AMCIS). Baltimore: Univ. of Baltimore, 1998. http://aisle.aisnet.org/amcis1998/325

15. Davis, F.D., Perceived usefulness, perceived ease of use, and user acceptance of information technology, MIS Quarterly, Vol.13, no. 3, pp.319-340, 1989. doi.org/10.2307/249008

16. Venkatesh, V.; Bala, H., Technology Acceptance Model 3 and a Research Agenda on Interventions, Decision Science, Vol. 39 , no. 2, pp. 273-312, 2008. doi.org/10.1111/j.1540-5915.2008.00192.x

17. V. Venkatesh, V., Determinants of perceived ease of use: integrating control, intrinsic motivation, and emotion into the technology acceptance model, Information Systems Research, Vol. 11, no.4, pp. 342-365, 2000. doi.org/10.1287/isre.11.4.342.11872_

18. Venkatesh, V.; Morris, M.G.; Davis, F.D.; Davis, G.B., User Acceptance of Information Technology: Toward a Unified View, MIS Quarterly, vol 27, no. 3, p.p. 425-478, 2003. doi.org/10.2307/30036540

19. Humphreys, T.E.; Murrian, M.J.; Narula, L., Deep-Urban Unaided Precise Global Navigation Satellite System Vehicle Positioning, IEEE Intelligent Transportation Systems Magazine, vol.12, no.3, pp. 109-122, 2020. doi: 10.1109/MITS.2020.2994121

20. Kassas,Z.Z.M.; Maaref, M.; Morales, J.J.; Khalife, J.J.; Shamei, K., Robust Vehicular Localization and Map Matching in Urban Environments Through IMU, GNSS, and Cellular Signals, IEEE Intelligent Transportation Systems Magazine, vol.12, no.3, pp. 36-25, 2020. doi: 10.1109/MITS.2020.2994110

21. I. Fernandez-Hernandez, I.; Senni, T.; Calle, D.; Cancela, S.; Vecchione, G.A.; Seco-Grandos, G., Analysis of High - Accuracy 
Satellite Messages for Road Applications, IEEE Intelligent Transportation Systems Magazine, vol.12, no.3, pp. 92-108, 2020. Doi. 10.1109/MITS.2020.2994074

22. Chen, H-H.; Chen, S-C., (2011), Applying the TAM to travellers' usage intentions of GPS devices, Expert Systems with Applications, vol. 38, Issue 5, pp. 6217-6221, 2011. [Online]. Doi.org./10.1016/j.eswa.2010.11.047.

23. E. Park, E.; Kim, K.J.; Jin, D.; del Pobil, A.P., Towards a successful mobile map services: An Empirical Examination of Technology Acceptance Model, International Conference on Networked Digital Technologies, pp. 420-428, 2012. Doi.org/10.1007/978/-3-642-30507-8_36

24. Nordhof, S.; Louw, T.; Innamaa, S.; Lehtonen, E.; Beuster, A.; Torrao, G.; Bjorvatn, A.; Kessel, T.; Malin, F.; Happee, R.; Merat, N., Using the UTAUT2 model to explain public acceptance of conditionally automated (L3) cars: A questionnaire study among 9.118 car drivers from European countries, Transportation Research Part F, vol. 74, pp. 280-297, 2020. doi.org/10.1016/j.trf.2020.07.015

25. Yang, L.; Bian, Y.; Zhao, X.; Liu, X.; Yao, X., Drivers' acceptance of mobile navigation applications: An extended technology acceptance model considering drivers' sense of direction, navigation application affinity and distraction perception, International Journal of Human-Computer Studies vol. 145, 2021. doi.org/10.1016/j.ijhcs.2020.102507

26. Aczel, A.D.; Sounderpandian, J., (2002). Complete Business Statistics. Boston, Mass: McGraw-Hill/Irwin, 2002.

27. GITD Raport, 2020, https://gitd.gov.pl/fileadmin/user upload/BTM/ZEZWOLENIA ZAGRANICZNE/2021/ Dane_statystyczne_dotyczace_transportu_miedzynarodowego_w_roku_2020.pdf (accessd: 2021-03-11).

28. Cortina, J.M, What is Coefficient Alfa? An Examination of Theory and Applications, Journal of Applied Psychology, vol.78, no.1, pp.98-104, 1993. [Online]. Availabe. doi.org/10.1037/0021-9010.78.1.98.

29. Bollen, K.A., Structural Equations with Latent Variables, Wiley, New York, 1989.

30. Pearl, J., "Causality”, New York: Cambridge University Press, 2000.

31. Byrne, B., Structural equation modelling with AMOS, Routledge, New York, 2010. [Online]. Available: doi.org/10.4324/9780203805534_

32. Weaver, B.; Wuensch,K.L., SPSS and SAS programs for comparing Pearson correlations and OLS regression coefficients. Behav Res, vol. 45, pp. 880-895, 2013. doi: 10.3758/s13428-012-0289-7

33. Żurek, M., Behavioral inclinations in financial markets in the light of SEMs, (In Polish), Torun: NCU Publishing House, 2016.

34. Efron, B.; Tibshirani, R., Bootstrap Methods for Standard Errors, Confidence Intervals, and Other Measures of Statistical Accuracy, Statistical Science, vol.1, pp. 54-75, 1986.

35. Freeman, C., Schumpeter's business cycles and techno-economic paradigms. In Reinert, E.S.; Drechsler, W.J.M.; C. Perez, C.; Kattel, E., (Eds.), Techno-economic paradigms: Essays in honour of Carlota Perez, 125-144. London; New York; Delhi: Anthem Press India, 2009 doi.org/10.7135/UPO9781843318224.010

36. Contractor, F.J., The world economy will need even more globalization in the post-pandemic 2021 decade, Jornal of International Businsess Studies, 2021. doi.org/10.1057/s41267-020-00394-y

37. Moscal, A., The Impact of Brexit on the European Union's Future Development in the Context of European Integration, Torun International Studies, vol. 1, no. 11, pp.25-36, 2018. [Online]. Available: http://dx.doi.org/10.12775/TIS.2018.002 